\title{
Poblaciones de diatomeas planctónicas en una represa subtropical : Embalse Río III, Argentina
}

\author{
Nora Gómez'
}

Palabras clave : Diatomeas, poblaciones, plancton, embalse.

En el presente trabajo se da a conocer la composición específica y la dinámica poblacional de las bacilariofitas halladas en el plancton limnético del Embalse Río III durante tres años y medio de muestreos. De los 32 taxones identificados las poblaciones más numerosas correspondieron a : Aulacoseira granulata, Aulacoseira granulata var. angustissima, Aulacoseira granulata var. angustissima f. spiralis, Aulacoseira lirata var. alpigena, Acinocyclus normanii $\mathrm{f}$. subsalsa y Cymbello affinis. La flora diatomológica hallada en la represa resultó ser alcalófila, eutrófica y oligohalobia. Este grupo algal en reiteradas oportunidades dominó el fitoplancton del embalse generalmente en períodos otonales y o invernales. La mezcla isotermal es uno de los principales condicionantes del incremento de las poblaciones de diatomeas en el embalse. Sin embargo $A$. granulata, que fue la bacilariofita más importante por su numerosidad, biomasa y persistencia en el tiempo, mostró desarrollos masivos durante dos periodos de estratificación térmica.

\section{Planktonic diatom populations in a subtropical reservoir : Embalse Rio III, Argentine.}

Keywords : Diatoms, population dynamic, plankton, reservoir.

This paper deals with the species composition and the population dynamics of the diatoms that were found in the limnetic plankton of the Embalse Rio III over tholl and a half years. 32 taxa were identified. The larger populations were : Aulacoseira lirata var. alpigena, Aulacoseira granulata, Aulacoseira granulata var, angustissima, Aulacoseira granulata var. angustissima f. spiralis, Actinocyclus normanii f. subsalsa and Cymbella affinis. The diatom flora found in Embalse Rio III were alkalophilous, oligohalobous eutróphic indicators. This algal group was often very dominant among the phytoplankton. This phenomenon usually took place during certain periods in autumn and winter. The isothermal mixing is one of the principal factors for the increase of the diatom populations of the reservoir. However $\boldsymbol{A}$. granulata was the dominant diatom in number, biomass and permanence; this alga shows massive development during the two periods of thermal stratification.

\section{Populations de Diatomées planetoniques dans un réservoir subtropical : Embalse Rio III, Argentine.}

Mots clés : Diatomées, dynamique de population, plancton, lac de barrage.

La composition du peuplement diatomique et la dynamique des diatomées planctoniques ont été suivies dans le réservoir Rio III pendant 3 ans et demi. 32 taxons, le plus souvent alcaliphiles, oligohalobes et indicateurs d'eurrophie ont été recensés. En automne et en hiver, les Diatomées les plus fréquentes Aulacoseira granulata, A. granulata var. angustissima, Aulacoseira granulata var. angustissima f. spiralis, Aulacoseira lirata var. alpigena, Actinocyclus normanii f. subsalsa et Cymbella affinis, dominent le phytoplancton. Dans ce lac de barrage, il se confirme que la période d'isothermie favorise la croissance des diatomées ; cependant, $A$. granulata, espèce la plus abondante à la fois par sa densité, sa biomasse et sa permanence, présente des développements massifs pendant les deux périodes de la stratification thermique.

1. Instituto de Limnologia « Dr. Raúl A. Ringuelet ». Casilla de Correo 712; La Plata, Buenos Aires, Argentina.

- Contribución $\mathbf{N}^{\circ} 463$ del Instituto de Limnologia « Dr Raúl A. Ringuelet ». 


\section{Introduccion}

De los grupos fitoplanctónicos, que pueblan el Embalse Rio III, las bacilariofitas desempeñan un papel primordial al ser causante de frecuentes floraciones. Por otra parte es reconocida la fidelidad de estas algas a determinadas variables ambientales (Lowe 1974), situación que las convierte en interesantes bioindicadores de los cambios que suceden en los ambientes que habitan. El conocimiento acerca de su composición especifica y de la dinámica de las poblaciones halladas en el plancton de la represa, objetivos de este trabajo, pretende contribuir a una mejor diagnosis y caracterización de este embalse. Las aguas del mismo son empleadas para la producción de energía hidroeléctrica, en la refrigeración de una central nuclear alli emplazada y con fines recreativos.

\section{Area de estudio. Material y metodos}

El Embalse Río III está situado à $32^{\circ} 10^{\prime}$ lat. S y $64^{\circ} 23^{\prime}$ long. W, en la Provincia de Córdoba. El mismo posee una superficie de 4529 ha, un volúmen de $560 \mathrm{Hm}^{3}$ y una profundidad máxima de 46,5 m (Fitz Simons 1938). Esta represa presenta estratificación térmica al final de la primavera y en verano, encuadrándose dentro de la categoría de « cálido- monomíctico » del esquema de Hutchinson \& Loffler (1956). En la tabla I se resumen las principales características físico-químicas del ambiente.

El muestreo se realizó a partir de cinco estaciones ubicadas a lo largo del eje mayor del embalse (fig. 1) y con un intervalo de dos meses entre noviembre de 1977 y mayo de 1981. Las muestras fueron obtenidas mediante bombeo.

Para el estudio de la variación horizontal de las bacilariofitas, de cada una de las cinco estaciones de muestreo se obtuvieron muestras integradas de la columna de agua comprendida entre superficie y los 20 metros, o hasta el fondo cuando la profundidad era menor a esta última. Con el propósito de obtener información acerca de la distribución vertical de estas algas, en la estación " $\mathrm{C}$ » se extrajeron muestras de los siguientes niveles : 0,$05 ; 1,0$; 2,$0 ; 5,0 ; 7,5 ; 10,0 ; 15,0$ y 20 metros, durante los dos primeros años de estudio.

Para el estudio detallado de los frústulos se empleó la metodología propuesta por Balech \&
Ferrando (1964) y por Hasle \& Syvertsen (1980), empleándose para el montaje definitivo del material Hyrax.

La cuantificación se realizó mediante el método de la pipeta (Kiselev 1969). Para el cálculo del volu. men celular, de las especies más numerosas, se asemejó la forma de cada uno de ellas a modelos geométricos (Vollenweider 1969) (Tabla II). En el caso de Aulacoseira granulata, especie que mostró un marcado polimórfismo (Gómez 1988) se calculó el volumen para cada uno de sus morfos, figurando en la Tabla II el mínimo y máximo obtenido.

Para la comparación de las estaciones de muestreo y de la numerosidad de las especies entre si se

Tabla I. Caracteristicas físico-quimicas del Embalse Río Il (Mariazzi et al., 1988).

\begin{tabular}{|c|c|c|c|}
\hline Variables & $\bar{x}$ & Minimo & Máximo \\
\hline Temperatura $\left({ }^{\circ} \mathrm{C}\right)$ & 16,7 & 7,7 & 25,5 \\
\hline Conductividad $\left({ }_{\mu} \mathrm{S} \mathrm{cm}^{-1}\right)$ & 140,0 & 86,0 & 193,0 \\
\hline $\mathrm{O}_{2}\left(\mathrm{mg} \mathrm{l}^{-1}\right)$ & 7,8 & 0,4 & 10,7 \\
\hline $\mathrm{C}-\mathrm{CO}_{3}=\left(\mathrm{mg} \mathrm{l}^{-1}\right)$ & 0,1 & 0,0 & 1,1 \\
\hline $\mathrm{C}-\mathrm{CO}_{3} \mathrm{H}^{-}$(mg l-1) & 15,8 & 10,1 & 22,9 \\
\hline $\mathrm{P}_{-} \mathrm{PO}_{3}^{-}\left(\mu \mathrm{g} \mathrm{l}^{-1}\right)$ & 7,7 & 0,0 & 93,0 \\
\hline $\mathrm{N}^{-\mathrm{NO}_{3}}\left(\mathrm{mg} \mathrm{l}^{-1}\right)$ & 0,08 & 0,00 & 0,48 \\
\hline 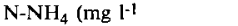 & 0,24 & 0,00 & 0,47 \\
\hline $\mathrm{Si}^{-\mathrm{SiO}_{2}}\left(\mathrm{mg} \mathrm{l}^{-1}\right)$ & 6,2 & 0,3 & 14,7 \\
\hline
\end{tabular}

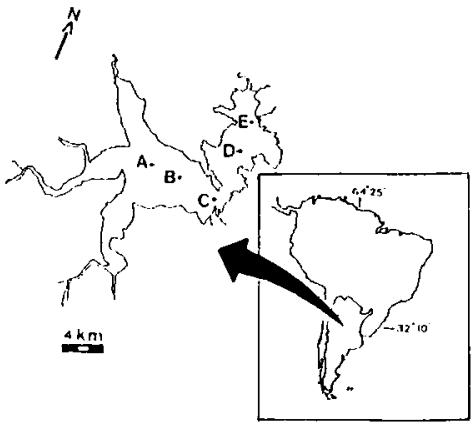

Fig. 1. Embalse Rio III : situación de las estaciones de muestreo. 
Tabla 11. Volumenes celulares calculados para las bacilariofitas más numerosas de la represa.

\begin{tabular}{|c|c|}
\hline & $\begin{array}{l}\text { Volumen } \\
{ }_{\mu} \mathrm{m}^{\prime} \text { cel }^{-1}\end{array}$ \\
\hline $\begin{array}{l}\text { Aulacoseira lirata } \\
\text { var. alpigena }\end{array}$ & 232 \\
\hline A ulacoseira granulata & $1479-10794$ \\
\hline $\begin{array}{l}\text { A. granuiata } \\
\text { var. angustissima }\end{array}$ & 414 \\
\hline $\begin{array}{l}\text { A. granulata } \\
\text { var. angustissima f. spiralis }\end{array}$ & 342 \\
\hline $\begin{array}{l}\text { Actinocyclus normanii } \\
\text { f. subsalsa }\end{array}$ & 7117 \\
\hline Cymbella affinis & 340 \\
\hline
\end{tabular}

aplicaron técnicas de análisis de agrupamiento, empleándose para ello el método de los grupos pares no ponderados aplicado sobre una matriz de similitud confeccionada a partir del coeficiente de correlación momento producto (Crisci \& Armengo) 1983).

\section{Resultados}

En el plancton limnético del Embalse Río III se identificaron las siguientes bacilariofitas:

\section{Orden : Centrales}

Aulacoseira lirata (Ehr.) Ross var. alpigena (Grunow) Haworth

Aulacoseira granulata (Ehr.) Simonsen

Aulacoseira granulata (Ehr.) var. angustissima (O. Müller) Simonsen

Aulacoseira granulata (Ehr.) var. angustissima f. spiralis (O. Müller) Czarnecki.

Cyclotella meneghiniana Kütz.

Melosira varians Ag.

Actinocyclus normanii (Greg.) Hustedt f. subsalsa

(Juhlin-Dannfelt) Hustedt

\section{Orden : Pennales}

Synedra ulna (Nitzsch) Ehr.

Cocconeis placentula Ehr, var. lineata (Ehr.) Van Heurk

Cocconeis placentula var. euglypta (Ehr.) Cleve

Cymbella affinis Kütz.

Cymbella cistula (Ehr.) Kirchner
Cymbella tumida (de Bréb.) Van Heurck

Gomphonema acuminatum Ehr.

Gomphonema subclavatum (Grun.) Grun. var. commutatum (Grun.) Mayer

Gomphonema truncatum Ehr.

Gomphonema truncatum var. capitatum (Ehr.)

Patrick

Gyrosigma acuminatum (Kütz.) Rabh.

Navicula cryptocephala Kütz.

Navicula cuspidata (Kütz.) Kütz.

Navicula notha Wallace

Pinnularia acrosphaeria Smith

Epithemia adnata (Kütz.) de Bréb. var. proboscidea (Kütz.) Patrick

Epithemia sorex Kütz.

Rhopalodia gibba (Ehr_) O. Müller

Nitzschia recta Hantz.

Nitzschia sigmoidea (Nitzsch) Smith

Nitzschia sinuata (W. Smith) Grun. var. tabellaria (Grun.) Grun.

Cymatopleura solea (de Bréb.) Smith

Cymatopleura solea var. gracilis Grun.

Surirella biseriata de Bréb.

Surirella tenera Gregory.

De la lista precedente $A$ ulacoseira lirata var. alpigena, A. granulata, A. granulata var angustissima, A. granulata var. angustissima f. spiralis, Actinocyclus normanii f. subsala y Cymbella affinis, fueron las mejor representadas numéricamente, en tanto que el resto presentó apariciones esporádicas y con escaso número de organismos.

\subsection{DISTRIBUCIÓN TEMPORAL}

En el transcurso de este estudio las bacilariofitas se convirtieron en reiteradas oportunidades en el grupo fitoplanctónico dominante. Esto se debió a los sucesivos desarrollos masivos producidos por distintas especies de este grupo algal, frecuentemente durante períodos otonales y/o invernales (fig. 2).

El comportamiento poblacional de las bacilariofitas más numerosas fue el siguiente :

- A. granulata fue la única diatomea que estuvo permanentemente presente en el plancton limnético del embalse. Las densidades poblacionales más importantes correspondieron a los meses de mayo de 1978 a 1981 , enero de 1979 y noviembre de 1980 . Esta última fecha fue la que reunió el valor más importante con $77861 \mathrm{cel} / \mathrm{m}^{2} .10^{6}$ (fig. 3). 
- A. normanii f. subsalsa sólo en marzo de 1980 no fue hallada en las muestras, correspondiendo el valor más elevado a julio de 1980 con 19620 cel $/ \mathrm{m}^{2} .10^{6}$ (fig. 7). Esta diatomea y $A$. granulata presentaron estados de resistencia, reconocibles por una condensación del citoplasma celular (Lund 1954 y Sicko-Goad et al. 1989). Estos estados vegetativos fueron observados en muestras de sedimento provenientes del fondo del embalse.

- A. lirata var. alpigena generalmente durante los muestreos de septiembre, noviembre y enero disminuyó su población, llegando en algunos casos a desaparecer. El desarrollo más notable lo alcanzó en mayo de 1979, con un máximo de 19843 $\mathrm{cel} / \mathrm{m}^{2} \cdot 10^{6}$ (fig. 6).

- A. granulata var. angustissima f. spiralis estuvo presente en el embalse desde enero hasta septiembre de 1978 y desde mayo de 1980 a mayo de 1981, registrándose en esta última fecha el máximo valor que fue de $13995 \mathrm{cel} / \mathrm{m}^{2} \cdot 10^{6}(\mathrm{fig} .5)$.

- A. granulata var. angustissima y Cymbella affinis desarrollaron poblaciones más pobres, con un máximo de $1193 \mathrm{cel} / \mathrm{m}^{2} .10^{6}$ (fig. 4) para la primera y de $38 \mathrm{cel} / \mathrm{m}^{2} .10^{6}$ para la segunda (fig. 8 ) mostrando ambas una presencia discontínua a lo largo del estudio.

En términos de volumen celular, como una medida cercana a la biomasa, $A$. granulata fue la más importante con una máximo de $91663 \mathrm{~mm} 3 / \mathrm{m}^{2}$ para noviembre de 1980. A. normanii f. subsalsa secundó a aquella especie con un registro de $78620 \mathrm{~mm}^{3} / \mathrm{m}^{2}$ en julio de 1980 . Si bien $A$. lirata var. alpigena mostró valores poblacionales elevados, como los de mayo y julio de 1979 , los mismos se vieron reflejados en valores relativamente bajos de volumen celular $\left(2740 \mathrm{~mm}^{3} / \mathrm{m}^{2}\right.$ y $1354 \mathrm{~mm}^{3} / \mathrm{m}^{2}$ respectivamente), como consecuencia del pequeño porte de esta diatomea (fig. 9).

Del dendrograma obtenido de la aplicación del coeficiente de correlación momento-producto (fig. 10) a la numerosidad promedio de las especies se evidencia una escasa similitud entre las fluctuaciones de las poblaciones en cuestión. Si bien los máximos poblacionales no se correspondieron temporalmente, hecho adjudicable a los distintos requerimientos ecológicos (Gómez 1988) y probablemente

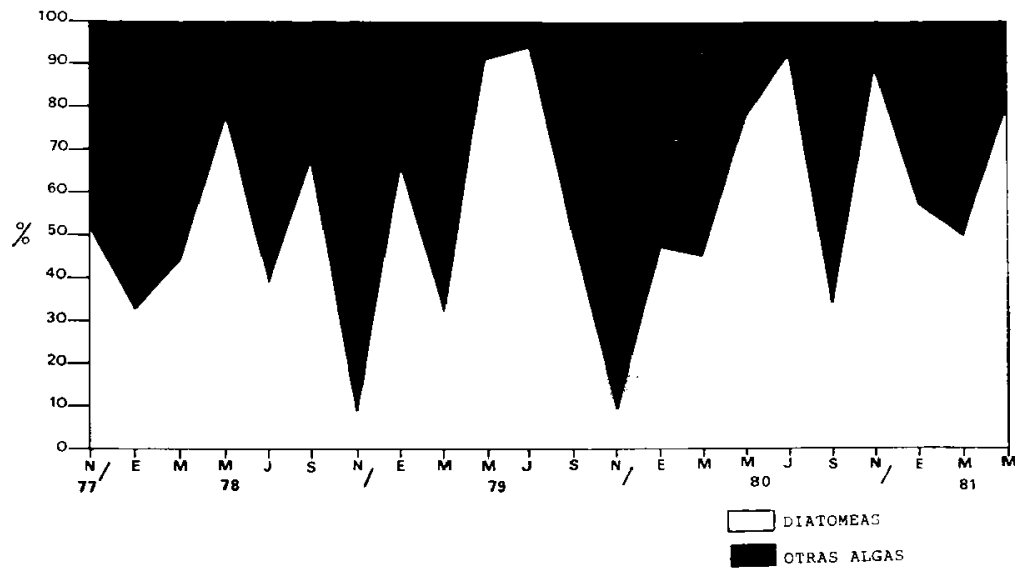

Fig. 2. Relaçión porcentual de la densidad de las diatomeas con otros grupos algales que habitan el Embalse Rio III. 

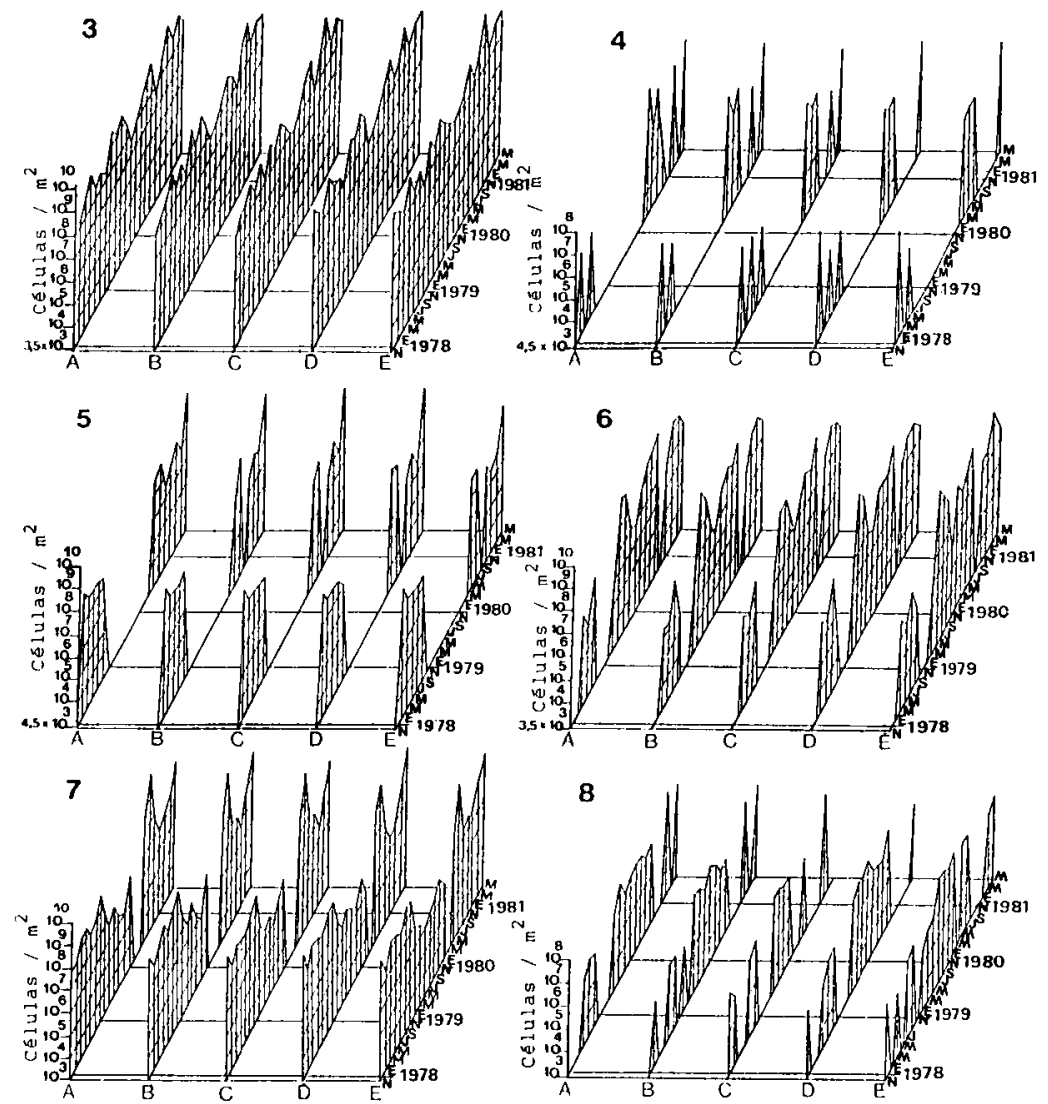

Fig. 3-8. Distribución espacio-temporal de : 3. Aulacoseira granulata ; 4. A. granulata var angustissima ; 5. A. granulata var. angustis. sima f. spiralis; 6. A. lirata var. alpigena; 7. Actinocyclus normanii f. subsalsa; 8 . Cymbella affinis. 


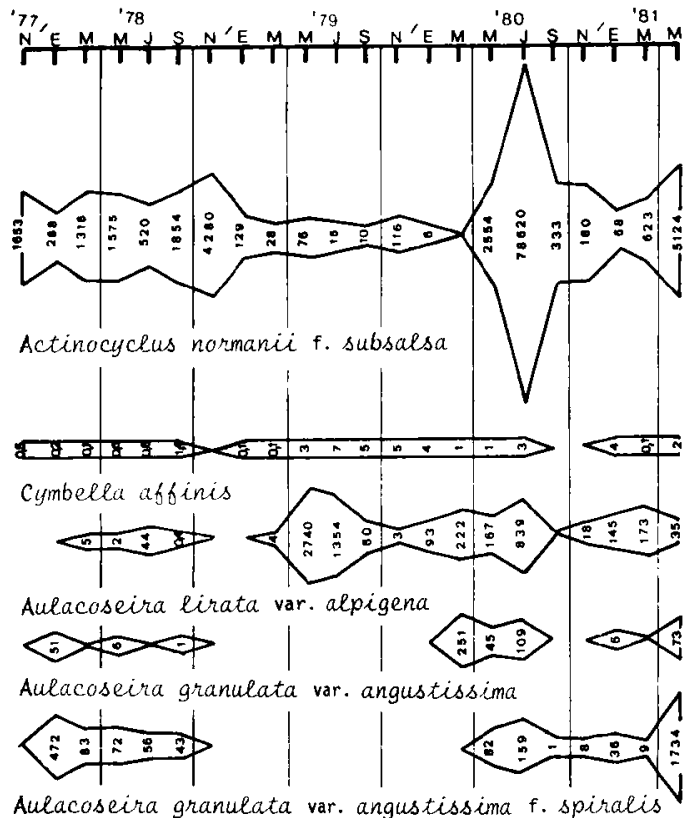

Aulacoseira granulata var. angustissima f. spiralis

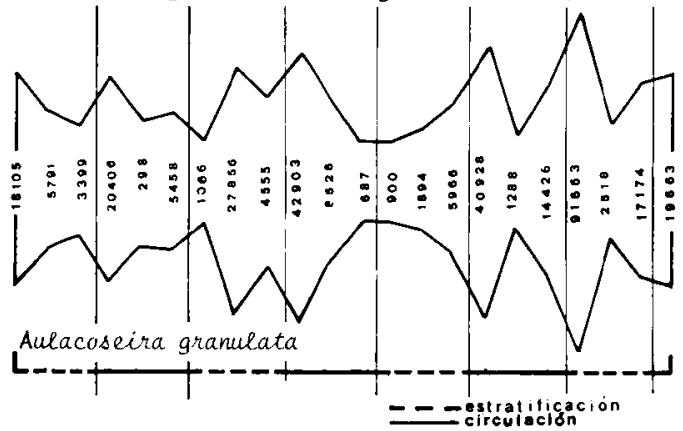

Fig. 9. Curvas esféricas representando la distribución temporal del biovolumen promedio $\left(\mathrm{mm}^{3} / \mathrm{m}^{1}\right)$ de las diatomeas dominantes y su relación con los períodos de estratificación y circulación del embalse. 


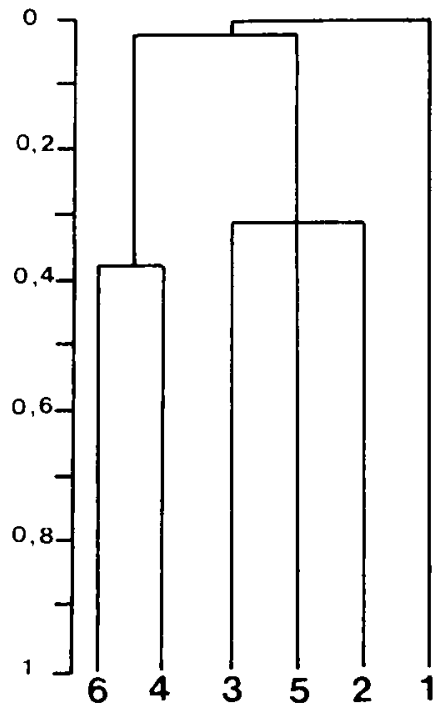

Fig. 10. Dendrograma obtenido a partir del coeficiente de correlación producto-momento en base a la densidad de : I. Aulacoseira granulata, 2. A. gramulata var. angustissima, 3. A. granulata var. angustissima f. spiralis, 4. A. lirata var. alpigena, 5. Actinocycius normanii f. subsolsa, 6. Cymbella affinis. UPGMA (ccc: 0.83).

a la competencia interespecifica, los mismos tuvieron lugar en distintos momentos de los periodos de circulación del embalse (fig. 9). A. granulata mostró además notables aumentos en su población durante la estratificación térmica de enero de 1979 y noviembre de 1980 ; estos desarrollos serian los determinantes de la segregación de esta especie en la gráfica.

\subsection{DISTRIBUCIÓN HORIZONTAL}

De acuerdo a los dendrogramas obtenidos, a partir de la numerosidad de las especies en las cinco estaciones de muestreo, se observó una manifiesta uniformidad que solo se interrumpió en noviembre de 1977 , enero y septiembre de 1978 y durante los meses de mayo, no evidenciándose una tendencia definida en estos agrupamientos (fig. 11). La heterogeneidad en la distribución horizontal de las diatomeas durante estos meses es atribuible a desarrollos locales más acentuados.

\subsection{Distribución VERTICAL}

En el Embalse Río III, durante los períodos de estratificación, se observa una mayor concentración de las distintas especies de diatomeas por encima de Ios niveles de marcado salto térmico ; como consecuencia de estas diferencias de temperatura se establecen picnoclinas que influencian notablemente la distribución irregular de estas algas en la columna de agua. En contraposición a esto último, durante la circulación isotermal, los perfiles tienden a ser uniformes debido a la mezcla que se impone en estos períodos (fig. 12).

\subsection{DIVERSIDAD}

Los valores de diversidad para las bacilariofitas, calculada a partir de la expresión de Brillouin (1956), estuvieron comprendidos entre 0,039 bits ind.-1 para noviembre de 1980 y 1,803 bits ind. $^{-1}$ en mayo de 1981 (fig. 13).

Los registros más bajos de este indice coincidieron con los desarrollos masivos más intensos de $A$. granulata que fueron los de enero de 1979 y de noviembre de 1980 y en una oportunidad con uno de los reiterados florecimientos del dinoflagelado Peridinium gatunense (Marzo 79).

En general la tendencia de este índice en el espacio mostró escasas variaciones y en lo que se refiere a su evolución en el tiempo manifestó generalmente incrementos durante los muestreos de otoño y/o invierno, coincidiendo con los períodos de mezcla.

\section{Discusión y conclusiones}

De los 32 taxones de diatomeas identificadas en el plancton limnético del embalse, las poblaciones más numerosas, a excepción de Cymbella affinis, pertenecieron al orden Centrales, siendo ellas: Aulacoseira granulata, $A$. granulata var. angustissima, $A$. granulata var, angustissima f. spiralis, $A$. lirata var. alpigena y Actinocyclus normanii f. subsalsa. El resto de las especies halladas, en su mayoría Pennales, presentaron apariciones esporádicas y con escaso número de organismos, resultado previsible por tratarse de muestras provenientes de la 

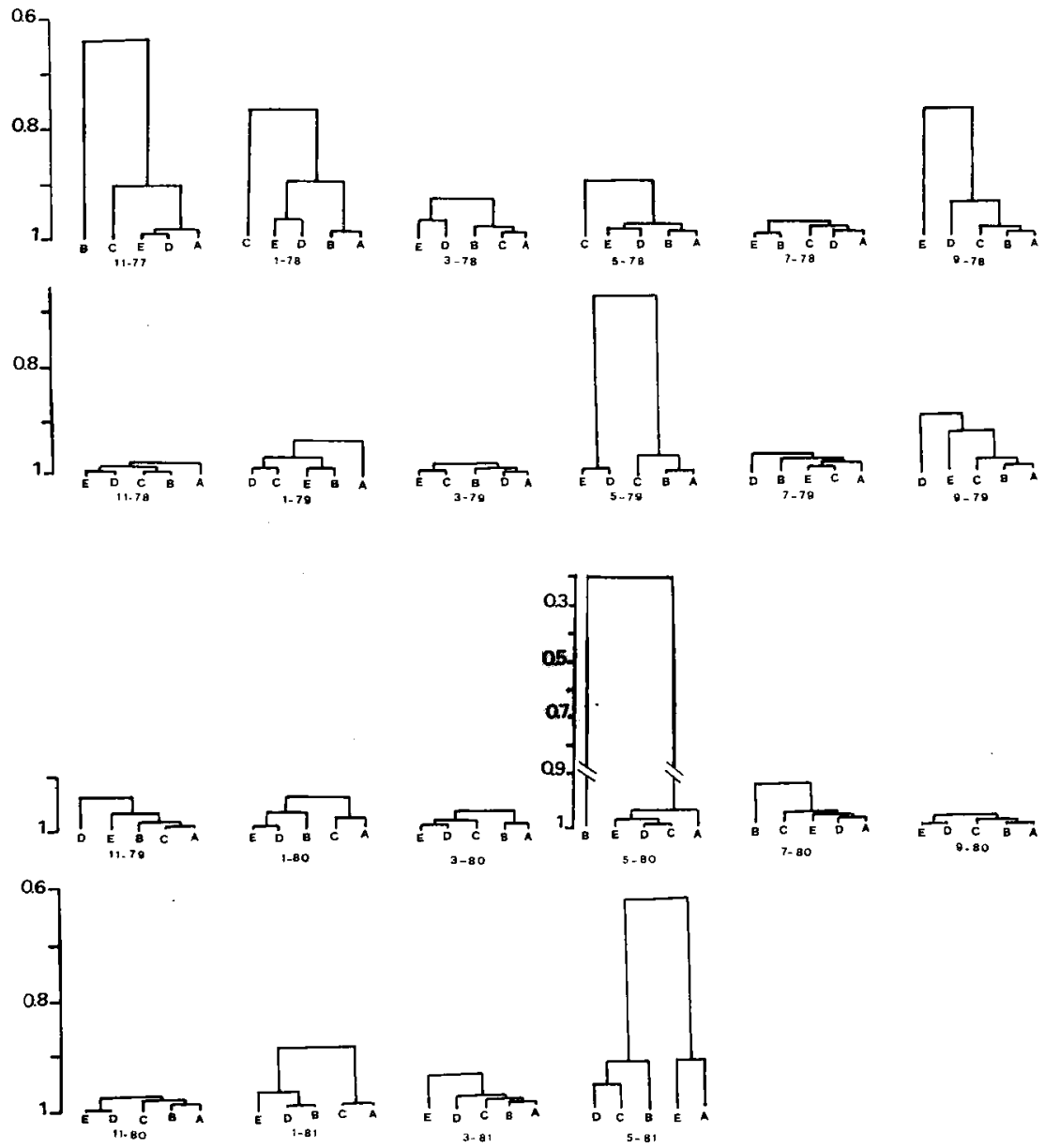

Fig. 11. Dendrograma resultante de la aplicación del coeficiente de correlación momento-producto a la numerosidad de las especies en las 5 estaciones de muestreo. UPGMA (cce: 0.72-0.99). 
(9)
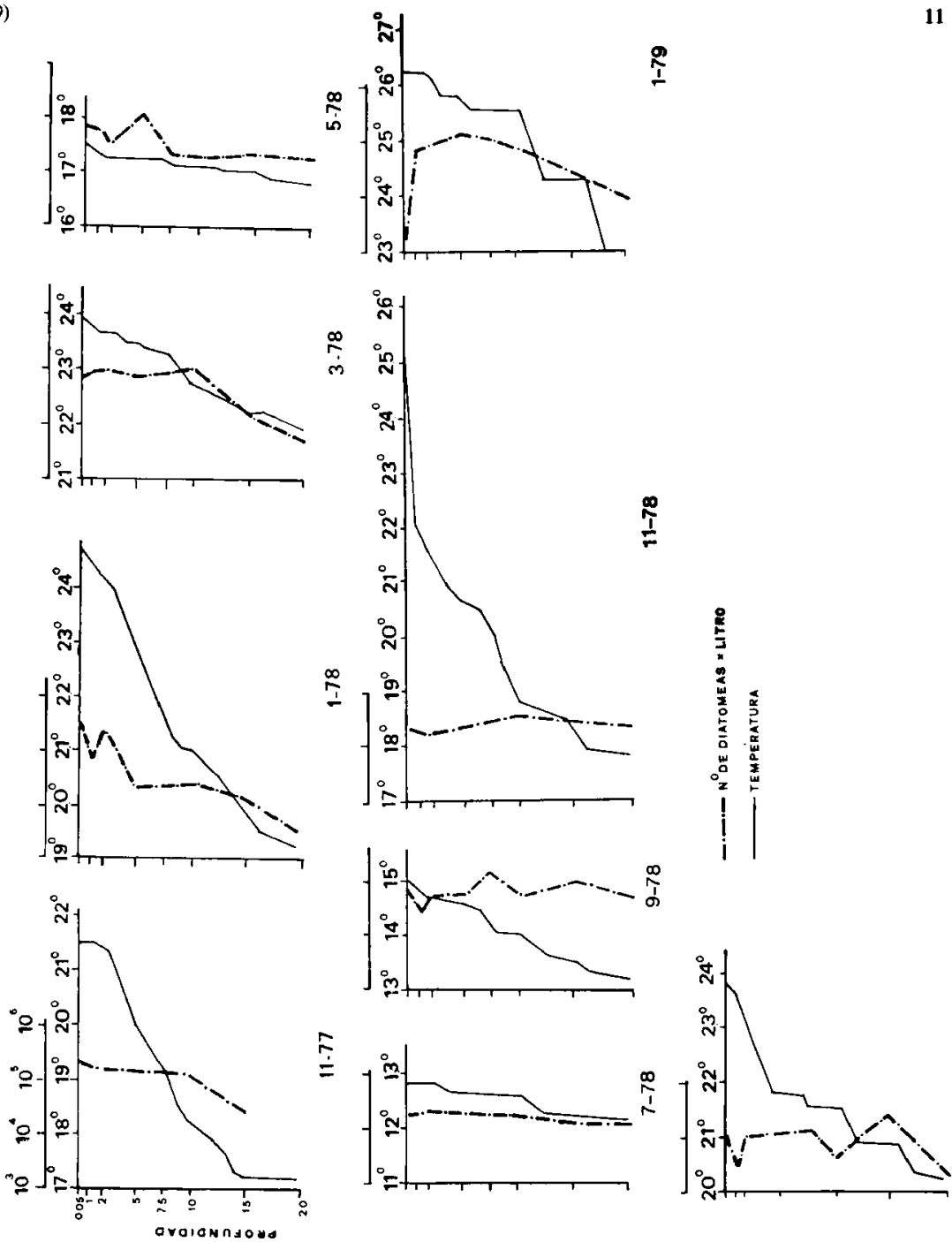

Fig. 12. Perfiles térmicos y distribución del número de diatomeas en relación con la profundidad. 


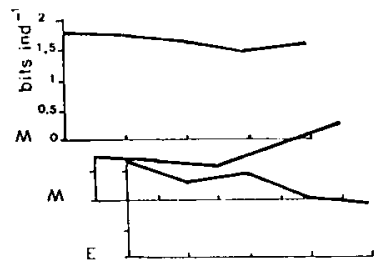

1981

N

$\mathrm{s}$

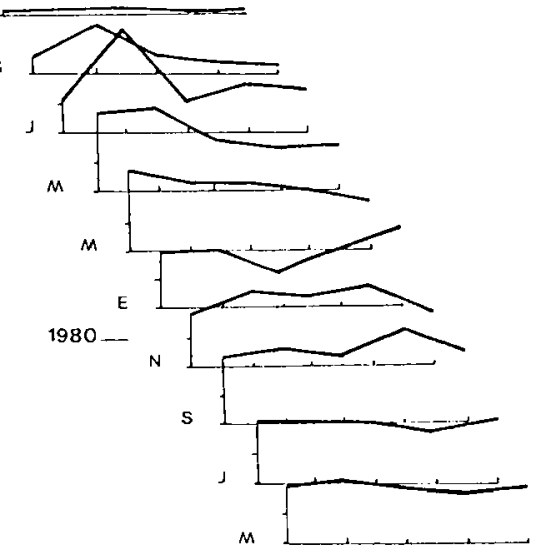

$M$
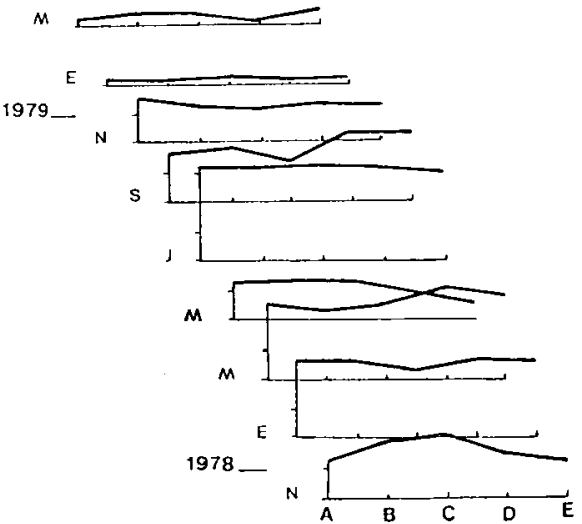

Fig. 13. Distribución espacio-temporal del indice de diversidad de Brillouin, calculado para las diatomeas del Embalse Rio III. 
zona limnética donde es reconocido el predominio de las diatomeas céntricas (Margalef 1983).

En el transcurso del presente estudiv las bacilariofitas se convirtieron en reiteradas oportunidades en el grupo fitoplanctónico dominante. Esto se debió a los sucesivos florecimientos, ocasionados por distintos representantes de este grupo tales como: $A$. granulata, A. granulata var. angustissima f. spiralis, $A$, lirata var. alpigena y $A$, normanii f, subsaisa que tuvieron lugar generalmente durante el otoño y/o invierno. El predominio de las bacilariofitas durante estas estaciones climáticas es un fenomeno recurrente en la represa, a juzgar por los resultados aquí obtenidos y los publicados por Bonneto et al. (1976).

El análisis de agrupamiento de especies demostró una débil similitud entre las poblaciones en estudio adjudicable a los distintos requerimientos ecológicos de las especies. Así pues según Gómez (1988) A. lirata var. alpigena dominó cuando la relación $\mathrm{Si} / \mathrm{P}$ fue elevada, prefiriendo baja luminosidad y alto nivel de agua embalsada. Por su parte $A$. normanii f. subsalsa se vió favorecida por una reducida relación $\mathrm{Si} / \mathrm{P}$ y $\mathrm{pH}$ elevados, en tanto Cymbella affinis incrementó su población con bajas temperaturas. El desarrollo de $A$. granulata estaría influenciado más por factores físicos que químicos ; la turbulencia generada durante la mezcla isotermal contribuye a resuspender los filamentos que permanecen en estado latente en los sedimentos, provenientes de ciclos anteriores. Probablemente los dos desarrollos masivos observados durante periodos de estratificación termal se hallan debido a cierta inestabilidad de esta última, hecho que suele ocurrir en el embalse (Boltovskoy \& Foggeta 1985), y que favorecería cierto grado de mezcla entre niveles próximos. Debido a la periodicidad de los muestreos no fue posible corroborar esta apreciación. La alta eficiencia fotosintética, la tasa intrínseca de caída y la posesión de estados de resistencia (Reynolds 1961) jugarian un rol fundamental en la notable repoblación de la masa de agua por parte de esta diatomea.

El establecimiento de picnoclinas a lo largo de la columna de agua favoreció la acumulación de diatomeas por encima de aquellos niveles, constituyendo el metalimnio un importante reservorio de diatomeas (Reynolds 1973). Por su parte la circulación isotermal favoreció una distribución más regular.
La distribución horizontal de las bacilariofitas en el embalse fue uniforme, a excepción de unas pocas ocasiones como consecuencia de desarrollos locales.

Los indices de diversidad mostraron escasas variaciones en el espacio y en lo referente a su distribución en el tiempo se observaron generalmente incrementos durante los periodos otonales $\mathrm{y} / \mathrm{o}$ invernales.

La flora diatomológica hallada en el plancton limnético del Embalse Río III se caracterizó por ser alcalófila, eutrófica y oligohalobia, confirmando las características ecológicas señaladas en la bibliografia (Patrick \& Reimer 1966, 1975, Luchini \& Verona 1972, Lowe 1974, Kilham \& Kilham 1975, Hasle 1977).

En el embalse las diatomeas proliferaron fundamentalmente durante la mezcla isotermal, coincidiendo con lo señalado por Reynolds (1973), Kilham \& Kilham (1975), Wetzel (1981), Toja Santillana (1984), Reynolds et al (1986) para otros cuerpos de agua. La sucesión de las poblaciones de bacilariofitas que pueblan esta represa estaría determinada por las exigencias ecológicas de las mismas y por el estatus competitivo individual que influenciarían el crecimiento potencial de las mismas, siendo factores determinantes en el predominio de una $u$ otra especie.

\section{Agradecimienıs}

La autora desea expresar su agradecimiento a la Dra. Martha Ferrario y al Lic. Andrés Boltovskoy por su colaboración en el desarrollo del presente estudio.

\section{Trabajos citados}

Balech (E.) \& Ferrando (H.J.). 1964. - Fitoplancton Marino. Eudeba, Buenos Aires: 151 p.

Boltovskoy (A.) \& Foggeta (M.). 1985. - Limnologia física del Embalse Rio III (Térmica, hidrología y derivaciones biologicas). Biol. Acuática. La Plata $7: 1-25$.

Brillouin (L.). 1956. - Science and information theory. Academic Press, New York.

Bomneto (A.A.), Di Persia (D.H.), Maglianesi (R.) \& Corigliano (M.C.). 1976. - Caracteres limnológicos de algunos lagos eutróficos de embalses de la región central de Argentina. Ecosur $3(5): 47-120$.

Crisci (J.V.) \& López Armengol (M.F.) 1983. - Introducción a la teoria y práctica de la taxonomia numérica. Monografias Científicas de la OEA, Serie Biología, Buenos Aires, $128 \mathrm{p}$.

Fitz Simons (S.E.). 1938. - Obras de embalse del Río III y Usina Hidroeléctrica de 15000 HP. Mir. Obr. Publ Nac, Dir. Gral. Irrig. Buenos Aires, 24 p. 
Gómez (N.). 1988. - Diatomeas del Embalse Rio HI. Tesis doctoral $N^{\circ} 508$, Facultad de Ciencias Naturales y Museo de La Plata, Buenos Aires.

Hasle (G.R.). 1977. - Morphology and taxonomy of Actinocyclus normanii f. subsaisa (Bacillariophyceae). Phycologia Is (3) : 321-328.

Hasle (G.R.) \& Syvertsen (E.E.). 1980. - The diatom genus Ceratulina : morphology and taxonomy. Bacillaria 3 : 79-113.

Hutchinson (G.E.) \& Löffler (H.). 1956. - The thermal classification of lakes. Proc. Nat. Acad. Sci. Washington 42 : 84-86.

Kitham (S.S.) \& Kilham (P.). 1975. - Melosira granulata, (Ehr.) Ralfs, morphology and ecology of a cosmopolitan freshwater diatom. Verh. Intern. Verein. Limnol, $19: 2716-2721$.

Kiselev (I.A.). 1969. - Plankion Morei I. Kontinentalnykh. Vodoimov. I. Nauka, Leningrad : 438 p.

Lowe (R.L.). 1974, - Environmental requirements and pollution tolerance of freshwoter diatoms. Nat. Envir. Res. Cent. Envir. Protec. Agency, Cincinati, Ohio, USA : 333 p.

Luchini (1.) \& Verona (C.A.). 1972. - Catálogo de las Diatomeas Argentinas. I. Diatomeas de aguas continemtales. Contrib. $\mathrm{N}^{\circ} 197 \mathrm{CIC}: 304 \mathrm{p}$.

Mariazzi (A.A.), Romero (M.C.) \& Conzonno (V.H.). 1988. Ciclo anual $y$ heterogeneidad de procesos autotróficos y heterotróficos, y caracteres fisicoquímicos del Embalse Río III, Córdoba, Argentina. Rev. Asoc. Cs. Nat. Litoral 19 (2): 167-181.
Margalef (R.). 1983. - Limnologia. Ed. Omcga, Barcelona : $1010 \mathrm{p}$.

Patrick (R.) \& Reimer (Ch. W.). 1966, - The diatoms of the United States. Vol. I. Monograph of the Academy of Nalural Sciences of Philadelphia. 13:688 p.

Patrick (R.) \& Reimer (Ch. W.). 1975. - The diatoms of United States. Vol. II. Monograph of the Academy of Natural Sciences of Philadelphia, 13:209 p.

Reynolds (C.S.). 1973, - The seasonal periodicity of plankton diatoms in a shallow Eutrophic Lake. Freshwat. Biol. 3 : 89.110.

Reynolds (C.S.), Montecino (M.E.), Graf (M.E.) \& Cabrera (S.). 1986. - Short-term dynamics of Melosira populations in the plankton in Central Chile. Journ. Plankton Res. 8 (4) : 715-740.

Sick o-Goad (L.), Stoermer (E.F.) \& Kociolek (J.P.P.). 1989. Diatom resting cell rejuvenation and formation : time course species records and distribution. Journal of Plankton Research (2) : 375-389.

Toja Santillana (J.). 1984, - Limnología de los embalses para el abastecimiento de aguas a Sevilla. Publ. Minist. Ob. Publ. Urban. España : 1-146.

Vollenweider (R. A.). 1969. - Primary Production in Aquatic Environments. IBP. Handbook 12, Blackwell. Sci. Oxford : $212 \mathrm{p}$.

Wetzel (R.G.) 1981. - Limnologia. Ed. Omega, Barcelona : $679 \mathrm{p}$. 